\title{
The Inviscid Burgers Equation with Initial Data of Brownian Type
}

\author{
Zhen-Su She $^{1}$, Erik Aurell ${ }^{2,3}$, and Uriel Frisch ${ }^{1,2}$ \\ Applied and Computational Mathematics, Princeton University, NJ 08544, USA \\ 2 CNRS, Observatoire de Nice, BP 229, F-06304 Nice Cedex 4, France \\ 3 Department of Mathematics, KTH, Stockholm, Sweden
}

Received February 21, 1992

\begin{abstract}
The solutions to Burgers equation, in the limit of vanishing viscosity, are investigated when the initial velocity is a Brownian motion (or fractional Brownian motion) function, i.e. a Gaussian process with scaling exponent $0<h<1$ (type $A$ ) or the derivative thereof, with scaling exponent $-1<h<0$ (type $B$ ). Largesize numerical experiments are performed, helped by the fact that the solution is essentially obtained by performing a Legendre transform. The main result is obtained for type $A$ and concerns the Lagrangian function $x(a)$ which gives the location at time $t=1$ of the fluid particle which started at the location $a$. It is found to be a complete Devil's staircase. The cumulative probability of Lagrangian shock intervals $\Delta a$ (also the distribution of shock amplitudes) follows a $(\Delta a)^{-h}$ law for small $\Delta a$. The remaining (regular) Lagrangian locations form a Cantor set of dimension $h$. In Eulerian coordinates, the shock locations are everywhere dense. The scaling properties of various statistical quantities are also found. Heuristic interpretations are provided for some of these results. Rigorous results for the case of Brownian motion are established in a companion paper by Ya. Sinai. For type $B$ initial velocities (e.g. white noise), there are very few small shocks and shock locations appear to be isolated. Finally, it is shown that there are universality classes of random but smooth (nonscaling) initial velocities such that the long-time large-scale behavior is, after rescaling, the same as for type $A$ or $B$.
\end{abstract}

\section{Introduction}

Burgers' equation

$$
\partial_{t} u+u \partial_{x} u=\nu \partial_{x x}^{2} u
$$

was introduced in the twenties as a model of turbulence $[1,2]$. The discovery of its integrability in the early fifties by Hopf [3] and Cole [4] has led to a rather complete understanding of the properties of individual solutions, particularly in the inviscid limit $(\nu \rightarrow 0)$ and also to the realization that... it is not a good model of turbulence, 
because it does not display any chaos, even when a force is added to the right-hand side.

More recently it has been discovered that Burgers equation arises naturally in a number of different problems. Zel'dovich [5] has proposed to use the multidimensional Burgers equation to study the formation of large-scale structures in the Universe. Kardar, Parisi, and Zhang [6] have shown that Burgers' equation can be used for studying the dynamics of interfaces, particularly when a random force is added. Kuramoto [7] has shown that the phase diffusion equation

$$
\partial_{t} \psi=\alpha \partial_{x x}^{2} \psi+\beta\left(\partial_{x} \psi\right)^{2}
$$

describes long wavelength phase modulations in a medium with travelling periodic waves (when parity-invariance holds). Observe that (2) is equivalent to Burgers' equation as long as $\alpha>0$, after $x$-differentiation and suitable rescaling. Among the many applications of phase diffusion, let us mention here the transmission of neural signals along giant axons of squid [8].

Thanks to the Hopf-Cole solution, there is a fairly good understanding of the properties of the solution to Burgers' equation in the inviscid limit when the initial velocity $u_{0}(x)$ is smooth. Typically, after some time, the solution $u(x, t)$ displays isolated discontinuities (shocks), separated by smooth regions, which for long times become ramps of slope $1 / t$. When the initial velocity is smooth and random (e.g., Gaussian with a spatially decreasing correlation function) there are few rigorous results, but various asymptotic laws have been proposed, based on partially heuristic arguments. They concern mostly the $k^{-2}$ behavior of the energy spectrum at high wavenumbers $k$ and the law of decay of the mean energy ([2, 9-11] and references therein).

It is our purpose here to investigate the behavior of solutions to the unforced Burgers' equation with random non-smooth initial velocities having no characteristic scale. The prototypes are the Brownian motion function and the white noise distribution, but we shall consider two broader classes (type $A$ and $B$ ) of which the former are instances. All our initial conditions can be characterized by a scaling exponent $h$ between -1 and $+1(h=1 / 2$ for Brownian motion and $h=-1 / 2$ for white noise; see Sect. 3 for precise definitions).

Such initial conditions arise naturally in at least two of the above mentioned problems. In extending Zel'dovich's work on the formation of "pancake structures" in the Universe, Gurbatov et al. [12] were led to use the (multi-dimensional) Burgers' equation in the inviscid limit with Gaussian initial conditions having a power-law spectrum with adjustable spectral exponent (see also [13-15] and the book [16]). In the neural signal transmission experiment of Musha et al. [8], the initial condition for the quantity which satisfies Burgers' equation is essentially white noise (at those scales where the phase diffusion equation applies).

Burgers' equation with an initial random velocity having scaling properties is an interesting problem in itself. For example one expects a conflict between the initially imposed scaling and the scaling which is dynamically generated through the formation of shocks. Finally, we discovered the existence of universality classes of random but smooth initial velocities such that the long-time large-scale behavior of the solutions (suitably rescaled) to Burgers' equation is the same as that arising after a finite time for the non-smooth initial conditions considered in this paper (see Sect. 7).

The present investigation is a coordinated effort with Ya. Sinai, who has been able to rigorously establish some of our conjectures. These conjectures were based on qualitative features of numerical solutions of Burgers' equation with an initial 
velocity field which is the Brownian motion function. Ya. Sinai obtained further rigorous quantitative results which we have numerically verified with high accuracy. The results by Ya. Sinai are presented in a separate paper in the same issue, hereafter referred to as the "companion paper" [17]. In the present paper, our approach will be a mixture of heuristic theory and of numerical experiments. The paper is organized as follows. In Sect. 2, we briefly recall the Hopf-Cole solution of Burgers' equation and define our notation. In Sect. 3, we introduce two types of Gaussian initial conditions with scaling properties. Type $A$ has scaling exponents $0<h<1$, and contains as a special case the Brownian motion for which exact results are established in the companion paper. Type $B$ has scaling exponents $-1<h<0$, and contains as a special case the white noise. We then establish some elementary scaling properties of the solution. The numerical strategy used for this study and related problems are presented in Sect. 4. In Sect. 5, we present qualitative aspects of the solutions together with heuristic interpretations. Section 6 is devoted to quantitative results such as the accurate determination of scaling exponents for various statistical quantities; some conjectures are made for initial conditions other than the Brownian motion. In the concluding Sect.7, we summarize key results and make various remarks.

\section{Hopf-Cole in a Nutshell}

Burgers' equation (1) may be rewritten as

$$
\partial_{t} u+\partial_{x}\left(\frac{u^{2}}{2}\right)=\nu \partial_{x x}^{2} u
$$

Let us introduce a potential function $\psi$, defined by $u=-\partial_{x} \psi$. Using the Hopf-Cole transformation $[4,3] \psi=2 \nu \ln \theta$, we obtain a heat equation for $\theta$ :

$$
\partial_{t} \theta=\nu \partial_{x x}^{2} \theta
$$

In the absence of boundaries, the solution of (4), for $t>0$, is

$$
\theta(x, t)=\frac{1}{\sqrt{4 \pi \nu t}} \int_{-\infty}^{\infty} e^{-\frac{(x-a)^{2}}{4 \nu t}} \theta_{0}(a) d a
$$

where

$$
\theta_{0}(a)=\exp \left[\frac{1}{2 \nu} \psi_{0}(a)\right]
$$

and $\psi_{0}(a)$ is the initial potential function such that $u_{0}(a)=-\partial_{a} \psi_{0}(a)$.

In the inviscid limit $(\nu \rightarrow 0)$, the solution found by a steepest descent argument reads

$$
\psi(x, t)=\max _{a}\left[\psi_{0}(a)-\frac{(x-a)^{2}}{2 t}\right], \quad \text { for } \nu \rightarrow 0 .
$$

We denote by $a(x, t)$ the location where the maximum in (6) is achieved (when it is unique). The function $a(x, t)$ will be called here the inverse Lagrangian function. As we shall see below, its inverse $x(a, t)$, is the usual Lagrangian function, i.e. the location at time $t$ of the fluid particle initially at $a$. Both functions, $x(a)$ and $a(x)$, will be central objects of interest in our study. In what follows, we shall define the solution of the inviscid Burgers equation by (6). The finiteness of the maximum is guaranteed as long as $\psi_{0}(a)$ increases less rapidly than $a^{2}$ for $a \rightarrow \infty$. 
We now show [2] that, for fixed $t$, the inverse Lagrangian function $a(x)$ is a non-decreasing function of $x$. Indeed, let $a_{*}$ and $a_{*}^{\prime}$ denote one of the maximizing locations corresponding to $x$ and $x^{\prime}$, respectively. We then have, by definition of the maximum,

$$
\psi_{0}\left(a_{*}\right)-\frac{\left(x-a_{*}\right)^{2}}{2 t} \geq \psi_{0}(a)-\frac{(x-a)^{2}}{2 t}, \quad \forall a
$$

and

$$
\psi_{0}\left(a_{*}^{\prime}\right)-\frac{\left(x^{\prime}-a_{*}^{\prime}\right)^{2}}{2 t} \geq \psi_{0}(a)-\frac{\left(x^{\prime}-a\right)^{2}}{2 t}, \quad \forall a
$$

We substitute $a=a_{*}^{\prime}$ into (7) and $a=a_{*}$ into (8), we add the resulting inequalities and multiply by $2 t$. After simplifications, we obtain

$$
2\left(x-x^{\prime}\right)\left(a_{*}-a_{*}^{\prime}\right) \geq 0 .
$$

This proves the result.

The maximizing condition in (6) can also be expressed as

$$
\psi(x, t)+\frac{x^{2}}{2 t}=\max _{a}\left[\phi_{0}(a)+\frac{x a}{t}\right], \quad \text { for } \nu \rightarrow 0,
$$

where $\phi_{0}(a)=\psi_{0}(a)-\frac{a^{2}}{2 t}$. Hence, the solution to Burgers' equation can be expressed in terms of a Legendre transform of $\phi_{0}(a)$. This gives a direct geometrical method for the construction of the solution. The key observation is that the Legendre transform of a function coincides with the Legendre transform of its convex envelop. When the initial condition has bounded velocity gradients, $\phi_{0}(a)$ is convex in $a$, for sufficiently short times [because $a^{2} / 2 t$ is large and convex, and $\psi_{0}(a)$ has bounded second derivatives]. For such times the Legendre transformation is smooth, and the maximum in (6) is obtained at the single Lagrangian location $a$ at which the derivative with respect to $a$ of the bracketed quantity vanishes. This gives

$$
x=a+t u_{0}(a) .
$$

Hence, $x$ is the location at time $t$ of a fluid particle starting at location $a$ and moving with velocity $u_{0}(a)$. Thus, it is justified to call $x$ and $a$, Eulerian and Lagrangian coordinates, respectively. By taking the $x$-derivative of (6), one easily finds that

$$
u(x, t)=u_{0}(a) .
$$

After some time, singularities may develop, corresponding to those $a$ 's where the convex hull of $\phi_{0}(a)$ departs from the function itself. For some values of $x$ there may be two values $a^{-}<a^{+}$where the maximum is achieved. For such values of $x$, the (Eulerian) velocity is discontinuous. From (11), its left and right limits are given respectively by

$$
\begin{aligned}
& u^{-}=\left(x-a^{-}\right) / t, \\
& u^{+}=\left(x-a^{+}\right) / t .
\end{aligned}
$$

Thus, the amplitude of the velocity discontinuity (shock) $u^{-}-u^{+}$is proportional to the length of the Lagrangian interval $a^{+}-a^{-}$which gets absorbed into the shock:

$$
u^{-}-u^{+}=\left(a^{+}-a^{-}\right) / t=\Delta a / t .
$$


Points within shock intervals such as $\left[a^{-}, a^{+}\right]$will be called Lagrangian shock points. The corresponding $x$ 's will be called Eulerian shock points.

Note that the quantity $\Delta a=a^{+}-a^{-}$may also be viewed as the mass of the fluid absorbed into the shock (assuming an initially uniform mass density).

Finally, we define Lagrangian regular points to be points $a_{*}$ such that they have not participated in a shock for any time $0 \leq t^{\prime} \leq t$. This is equivalent to the geometrical statement that there exists a couple $\left(x_{*}, C_{*}\right)$ such that the parabola $a \mapsto\left(a-x_{*}\right)^{2} /(2 t)+C_{*}$ touches the graph of $a \mapsto \psi_{0}(a)$ at the single point $\left(a_{*}, \psi_{0}\left(a_{*}\right)\right)$. The point $x_{*}$ is said to be an Eulerian regular point.

Concerning regular points, there are several interesting possibilities. (i) If the function $\psi_{0}(a)$ is differentiable once, the tangent at $a_{*}$ is unique and this implies the uniqueness of the parabola. (ii) If the function $\psi_{0}(a)$ is twice differentiable, there may be an entire interval of regular points near $a_{*}$. (iii) If, however, the function $\psi_{0}(a)$ is not differentiable at all, the parabolas may not be unique and generally there will be a whole range of $x_{*}$ 's for a given $a_{*}$. Case (ii) is standard for very smooth initial data. Case (i) and (iii) will be relevant for the type $A$ and type $B$ non-smooth initial velocities which are considered here.

\section{Self-Similar Initial Conditions and Elementary Scaling Properties of the Solution}

We now specify the different types of initial conditions chosen for our investigation. In all cases, the initial velocity $u_{0}(a)$ is a Gaussian random function with zero mean value. The velocity increments are homogeneous and self-similar. The former means that the statistical properties of increments are invariant under translations. The latter means that there is a scaling exponent $h$ such that

$$
u_{0}\left(a^{\prime}+\lambda a\right)-u_{0}\left(a^{\prime}\right) \stackrel{\text { law }}{=} \lambda^{h}\left(u_{0}\left(a^{\prime}+a\right)-u_{0}\left(a^{\prime}\right)\right) \quad \forall \lambda>0, \forall a, \forall a^{\prime},
$$

where $\stackrel{\text { law }}{=}$ refers to equality "in law," meaning that the 1.h.s. and r.h.s. have the same probability distribution.

The simplest instance is the Brownian motion function $b(a)$, a Gaussian random function satisfying $b(0)=0$ and $\left\langle b(a) b\left(a^{\prime}\right)\right\rangle=\inf \left(a, a^{\prime}\right)$. It is easily checked that for Brownian motion $h=1 / 2$. More generally, we will consider the class of fractional Brownian motions with $1>h>0$. We call these initial data type $A$ initial conditions.

Our second class of initial conditions, type $B$, are just the derivatives of the former. In other words, it is now the initial potential function $\psi_{0}(a)$ which is of type $A$. Type $B$ initial conditions for the velocity are not random functions but random distributions. They have scaling exponents $-1<h<0$. The simplest instance, corresponding to $h=-1 / 2$, is the white noise which is the derivative of the Brownian motion.

Both type $A$ and $B$ initial conditions can be characterized by their energy spectrum, which is easily seen to be a power-law:

$$
E(k) \propto|k|^{-1-2 h},
$$

where $k$ is the wavenumber.

The self-similarity of the initial conditions has an important consequence for the solutions to Burgers' equation, given by (6). To derive this relation, we start from (16) and specialize to $a^{\prime}=0$. We then integrate once to obtain a relation for the initial potential function:

$$
\psi_{0}(\lambda a) \stackrel{\text { law }}{=} \lambda^{1+h} \psi_{0}(a)
$$


Straightforward manipulations of (6), using (18) lead to:

$$
\psi\left(\lambda x, \lambda^{1-h} t\right) \stackrel{\text { law }}{=} \lambda^{1+h} \psi(x, t) \quad \forall \lambda>0 .
$$

In particular,

$$
\psi(x, t) \stackrel{\text { law }}{=} t^{\frac{1+h}{1-h}} \psi\left(x t^{-\frac{1}{1-h}}, 1\right)
$$

Similarly, for the velocity, we obtain:

$$
u(x, t) \stackrel{\text { law }}{=} t^{\frac{h}{1-h}} u\left(x t^{-\frac{1}{1-h}}, 1\right) .
$$

Such scaling relations allow us to express the (single-time) statistical properties of the solution at any time $t>0$ in terms of the solution at $t=1$. Hereafter, we shall set $t=1$. Note that for smooth (non-scaling) initial conditions, the statistical properties of the solution at two different times cannot in general be related. This is consistent with the observation that, for smooth initial conditions, it takes some time for shocks to be formed (typically the inverse of the largest initial negative velocity gradient). In contrast, for our non-smooth initial conditions, shocks are formed after an arbitrarily short time.

Note that the restriction on scaling exponents (e.g. $h<1$ ) guarantees that the solution given by (6) is almost surely finite. Indeed, for large $a$ 's the initial potential $\psi_{0}(a)$ grows as $|a|^{1+h}$ which is bounded by $a^{2}$ for $h<1$. Thus, the subtraction of the parabolic term $(x-a)^{2} / 2 t$ guarantees almost surely a bounded maximum achieved at one or several Lagrangian locations $a$.

\section{Discrete Numerical Analysis}

Explicit numerical realization of our non-smooth initial data requires discretization. That is, we have to introduce a smallest length $\Delta x$ (mesh size), and the solution of (6) should be studied at scales $\gg \Delta x$. In order to verify that the numerical solutions reported hereafter are representative of the continuous limit $(\Delta x \rightarrow 0)$, we have performed computations with different mesh sizes.

We have used two methods for generating initial data: a random walk method and a Fourier transform method. It is well known that a random walk with zero-mean and finite variance steps (and otherwise arbitrary distributions) converges, after rescaling, to the Brownian motion function at scales larger compared to the step size. Thus, we can generate a discrete Brownian motion function (of step size $\Delta x$ ) by sums of the form:

$$
b(M \Delta x)=\sum_{i=1}^{M} \omega_{i}
$$

where the $\omega_{i}$ 's are randomly and independently chosen with, e.g. a Gaussian probability distribution function, zero-mean and variance $\sigma^{2}$. We impose $\sigma^{2}=O(\Delta x)$ to ensure that $b(x)$ converges to the Brownian motion function as $\Delta x \rightarrow 0$. The corresponding discrete initial velocity we use is

$$
u_{0}^{(b)}(M \Delta x)=b(M \Delta x)
$$


where the superscript $(b)$ refers to "Brownian motion." For the initial potential function, we must integrate once. Given the lack of smoothness, no accuracy is lost by simply taking Riemann sums. Thus,

$$
\psi_{0}^{(b)}(M \Delta x)=-\sum_{i=1}^{M} u_{0}^{(b)}(i \Delta x)
$$

For the case where the initial velocity is white noise, we work directly with the potential, which is then the Brownian motion. We thus take:

$$
\psi_{0}^{(w)}(M \Delta x)=b(M \Delta x),
$$

where the superscript $(w)$ refers to "white noise."

The random walk method for generating initial conditions works only because Brownian motion is a Markov process. This is not the case for fractional Brownian motion which must be generated differently. Approximations to fractional Brownian motion with a finite large period $L$ can be generated as a Fourier series

$$
u_{0}(a)=\sum_{k} u_{k} e^{i k a}, \quad k=\frac{2 \pi n}{L} \quad(n=0, \pm 1, \pm 2, \ldots)
$$

where the $u_{k}$ 's are complex Gaussian random variables with variance $\left\langle\left|u_{k}\right|^{2}\right\rangle \propto$ $|k|^{-1-2 h}$, chosen independently except for the Hermitian symmetry $u_{k}=u_{-k}^{*}$. The velocity generated in this way is self-similar with exponent $h$ (at scales large compared to the mesh and small compared to $L$ ). The potential function can be similarly synthesized from its Fourier series. In practice, we generate directly the initial potential function with similarity exponent $1+h$, and use it to obtain the solution by solving (6).

Solving (6) with $t=1$ is done by searching for those $a$ 's which maximize the righthand side of (6). Given $\psi_{0}(a)$ at $N$ discrete points, determining $\psi(x, t)$ at all $N$ points apparently requires $O\left(N^{2}\right)$ operations, since searching for maximums for each point $x$ needs $O(N)$ operations. Actually, using the monotonicity of the Lagrangian function $x(a)$ [or its inverse $a(x)$ ] from (9), the total number of operations can be reduced to $O(N \log N)$ operations as we now show. Consider $N$ discrete points $x_{i}$, with $i=$ $1, \ldots, N$, and denote the corresponding maximizing $a$ 's by $a_{i}, i=1, \ldots, N$. First, we determine a $a_{N / 2}$ by searching through $x_{1}$ to $x_{N}$ [this requires $O(N)$ operations]. The next step is to determine $a_{N / 4}$ and $a_{3 N / 4}$, for which the search needs to be performed only in the subinterval $\left[x_{1}, a_{N / 2}\right]$ and $\left[a_{N / 2}, x_{N}\right]$. Thus, a total of $O(N)$ operations can determine two values of $a$. The third $O(N)$ operations gives then four maximizing $a$ 's, and so on. This greatly reduces the computational work for problems having large $N$ 's as is the case in the present study.

The numerical strategy discussed above gives a discrete version of the original continuous problem that we study. Thus, it is important in the numerical solution to be able to identify those features which are not an artefact of the discretization. In the discrete problem, there is generically only one point $a$ which maximizes the r.h.s. of (6). Thus the inverse Lagrangian function $a(x)$ obtained from the discrete problem is single-valued instead of being multi-valued at shocks, as happens in the continuous limit. The discrete analog of a shock is a change in $a$ occurring over one mesh size $\Delta x$, that is, $a\left(x_{i+1}\right)>a\left(x_{i}\right)$. Actually, we must require the stronger condition that

$$
a\left(x_{i+1}\right)-a\left(x_{i}\right) \gg \Delta x,
$$


so as to have a finite change in $a$ when the mesh $\Delta x$ is very small. Observe that, because the Burgers dynamics corresponds to an infinitely compressible fluid, Lagrangian intervals are infinitely compressed during the formation of shocks. It follows from (15) that, when (27) holds, the velocity displays a finite negative discontinuity. We shall call such points shock points. The amplitude of the jump, $\Delta a=a_{i+1}-a_{i}$, correspond physically to a Lagrangian interval $\left[a_{i}, a_{i}+\Delta a\right]$ which, at time $t$, is absorbed (compressed) into the shock.

Now, consider the opposite situation, where

$$
a\left(x_{i+m}\right)=a\left(x_{i}\right), \quad m \gg 1 .
$$

This corresponds to an initial point $a$ being stretched out infinitely to an (Eulerian) interval $\left[x_{i}, x_{i}+m \Delta x\right]$. This is how we identify regular points.

\section{Qualitative Features of the Solutions and Interpretation}

To gain a first insight into the property of the solutions, let us consider graphs of the velocity $u(x, 1)$ and the inverse Lagrangian function $a(x, 1)$. We recall that it is enough to study the property for a particular time, here $t=1$ (see Sect. 3). Figures 1
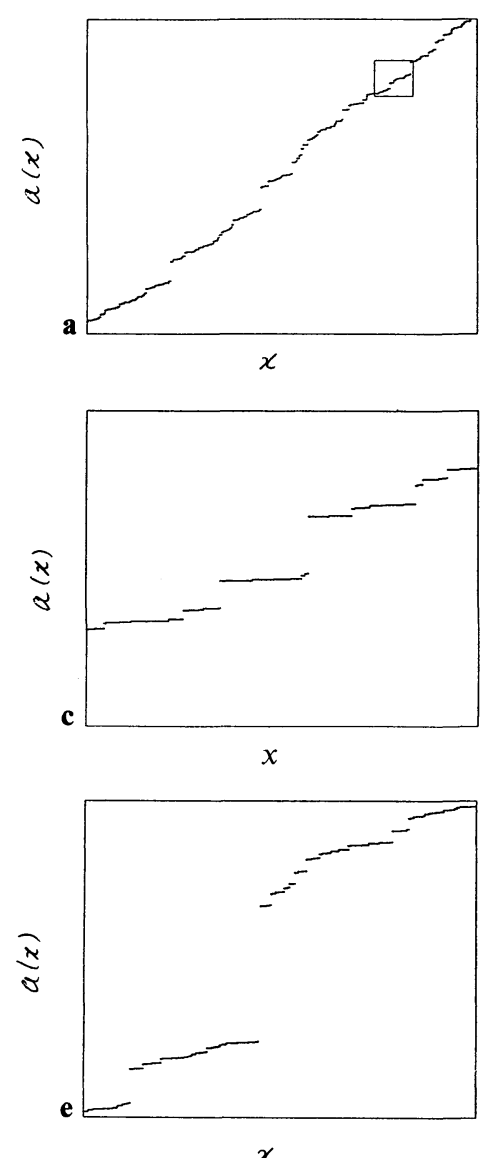
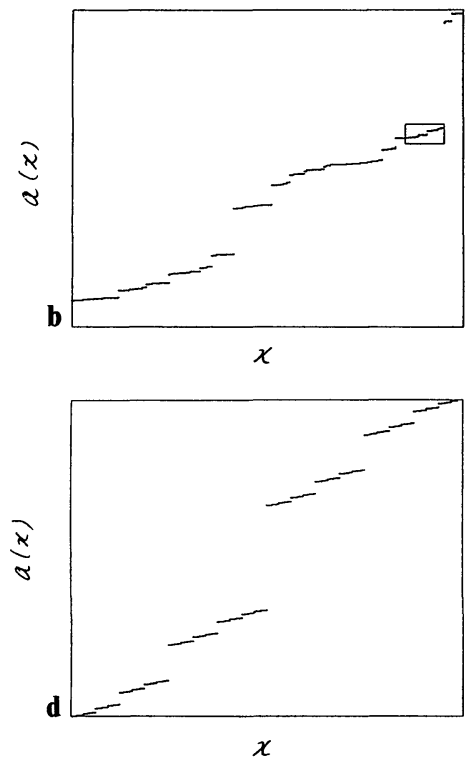

Fig. 1. a Inverse Lagrangian function $a(x)$ corresponding to the solution to the inviscid Burgers equation at $t=1$ with the Brownian motion function as initial velocity (type $A$ ). Note the hierarchy of plateaus and jumps. b, c Show successive zooming, displaying smaller and smaller structures. d Shows the (inverse of the) standard Devil's staircase constructed on the standard Cantor set, and e shows one of its randomized versions 

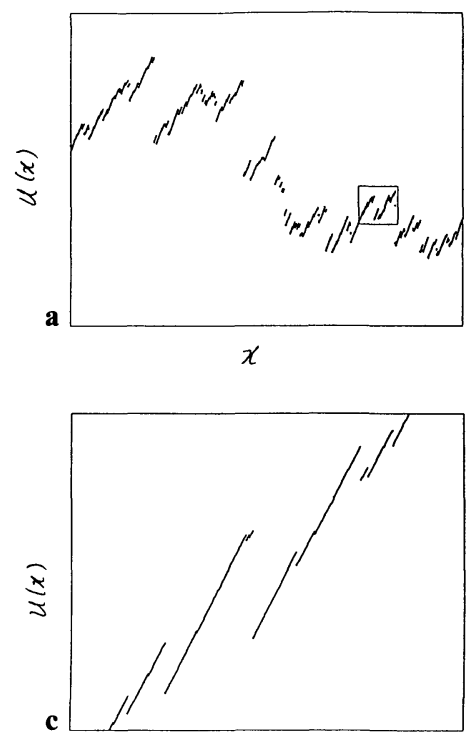

$x$

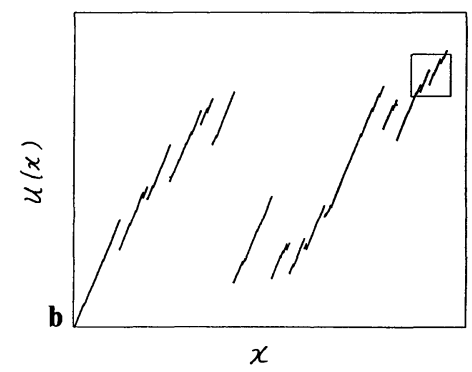

Fig. 2. a Eulerian velocity $u(x)$, solution to the inviscid Burgers equation at $t=1$ with the Brownian motion function as initial velocity (type $A$ ). b, c Show successive small-scale zooming, as in Fig. 1. Note the hierarchy of ramp-like structures with slope 1 and the proliferation of small shocks

and 2 illustrate such solutions, computed numerically from (6) with $N=100,000$ and $\Delta x=0.002$. Brownian motion is assumed for the initial velocity. Successive zooming has been set to display smaller and smaller structures. Let us first describe the inverse Lagrangian function $a(x)$. It displays a number of jumps which correspond to Lagrangian shock intervals. There are also a number of intervals where the function is seemingly flat (constant). However, when inspected more closely (after zooming), these intervals reveal smaller jumps inside. This scenario continues, until zooming reaches scales comparable to the mesh size.

The graph for $a(x)$ looks impressively like a Devil's staircase (in inverted coordinates). For comparison, in Fig. 1(d), we plot the graph of (the inverse of) the standard Devil's staircase function constructed on the Cantor set (by the $2 / 3$ rule). In Fig. 1(e), we plot also the graph of (the inverse of) a randomized version of the standard Devil's staircase. (Randomizing means that we shift randomly the position of jumps.) Observe that Fig. 1(a) and Fig. 1(e) have similar features. This observation has led us to conjecture that the Lagrangian function $a(x)$ for the solution to the Burgers equation with Brownian motion initial velocity is a Devil's staircase.

Now we turn to the velocity $u(x)$ (evaluated as always at $t=1) . u(x)$ is related to $a(x)$ by (12)-(15). As shown in Fig. 2, it displays a hierarchy of saw-tooth structures. There is an appearance of ramps with slope 1 , associated with (nearly) flat regions of $a(x)$. (The slope would be $1 / t$ at a time $t \neq 1$.) However, when zooming into these ramps, it is found that they actually contain many tiny shocks. This suggests that (in the continuous limit) for Brownian motion initial velocity, the total number of shocks per unit length is infinite and the Eulerian shock points are dense.

Let us now consider a particular type $B$ initial velocity, the white noise ( $h=$ $-1 / 2$ ). In Figs. 3 and 4, we show the graphs of the inverse Lagrangian function $a(x)$ and of the Eulerian velocity $u(x)$, displayed in the same way as in Figs. 1 and 2 . Here, we again see a hierarchy of shocks, but this time there are very few small shocks. Inspection of the largest magnifications (Figs. 3c and 4c) suggests that (in the continuous limit) for white noise initial velocity, both Euclidean shock points and 
Lagrangian regular points are isolated. In particular, the total number of shocks per unit length is now finite.

The two most conspicuous features for type $A$ initial data are (i) the presence of ramp-like structures with slope $1 / t$ (which holds also for type $B$ ); (ii) the denseness of shocks. The corresponding rigorous statements and their proofs are found in the
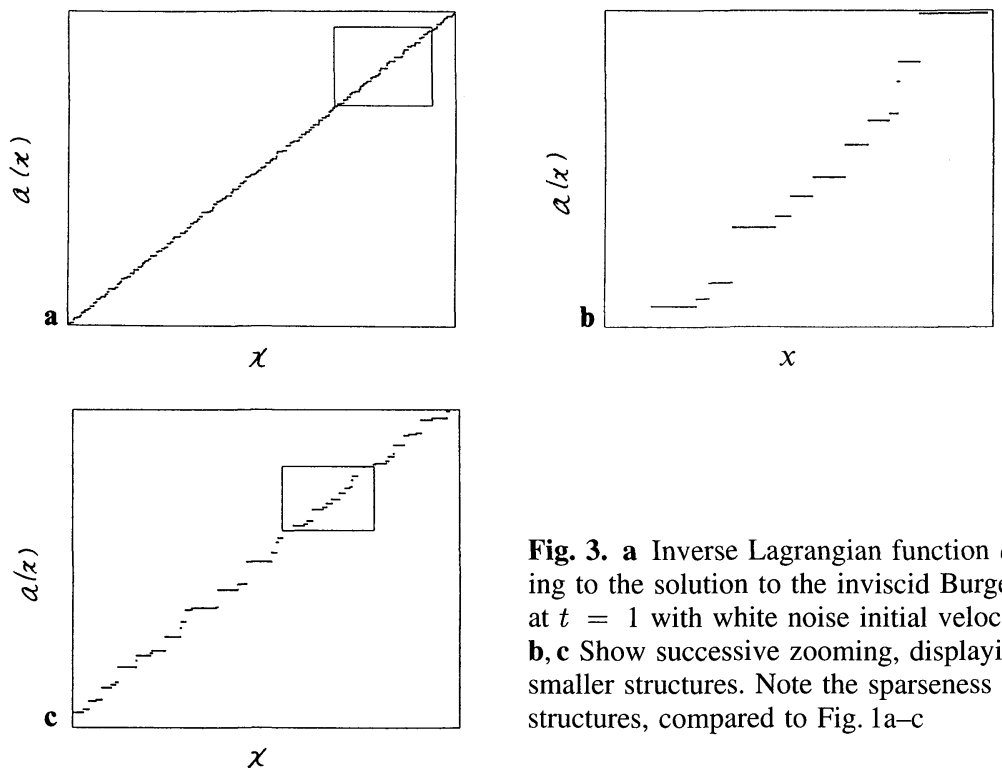

Fig. 3. a Inverse Lagrangian function $a(x)$ corresponding to the solution to the inviscid Burgers equation at $t=1$ with white noise initial velocity (type $B$ ). b, c Show successive zooming, displaying smaller and smaller structures. Note the sparseness of small scale structures, compared to Fig. 1a-c
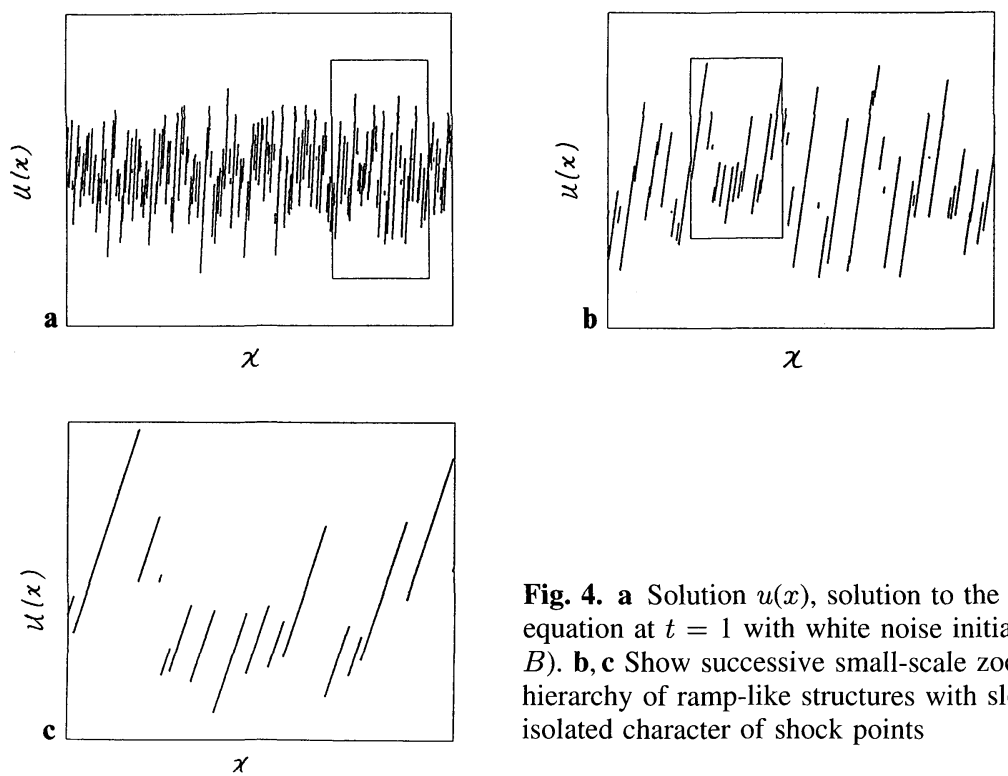

Fig. 4. a Solution $u(x)$, solution to the inviscid Burgers equation at $t=1$ with white noise initial velocity (type $B)$. b,c Show successive small-scale zooming. Note the hierarchy of ramp-like structures with slope 1 and the isolated character of shock points 
companion paper for the special case of Brownian motion initial velocity [17]. Here, we present some simple heuristic explanations.

We begin with the $1 / t$ ramps. They are associated with Lagrangian regular points. At such points the solution of the inviscid Burgers equation may be written implicitly as

$$
u(x, t)=u_{0}(a)
$$

where

$$
x=a+t u_{0}(a) .
$$

Let us now pretend that the same relation holds also for points $x^{\prime}$ close to $x$. Let $a^{\prime}$ denote the initial position associated with $x^{\prime}$. From (30) we then have

$$
x-x^{\prime}=a-a^{\prime}+t\left[u_{0}(a)-u_{0}\left(a^{\prime}\right)\right] .
$$

Let us first consider type $A$ (fractional Brownian motion) initial conditions. We then have for small $\left|a-a^{\prime}\right|$,

$$
\left|u_{0}(a)-u_{0}\left(a^{\prime}\right)\right| \sim\left|a-a^{\prime}\right|^{h}, \quad 0<h<1 .
$$

It follows that, for small $\left|a-a^{\prime}\right|$, the first term on the r.h.s. of (31) is negligible compared to the second. The derivation is completed by using (29), which implies

$$
\frac{u(x, t)-u\left(x^{\prime}, t\right)}{x-x^{\prime}} \simeq \frac{1}{t}
$$

For type $B$ initial data, the derivation is the same, except for the fact that $h$ is now negative, so that on the r.h.s. of (31) the second term dominates even more.

The same heuristic argument can be used to show that the inverse Lagrangian function $a(x)$ for type $A$ initial velocity $(0<h<1)$ has typically a vanishing derivative. Indeed, using (31) and (32), we have, for small $\left|a-a^{\prime}\right|$,

$$
\left|a-a^{\prime}\right| \sim\left|x-x^{\prime}\right|^{1 / h} .
$$

The latter is $o\left(\left|x-x^{\prime}\right|\right)$ since $1 / h>1$, thereby implying the vanishing of the derivative $\partial a / \partial x$. Actually, it is proven in the companion paper that for $h=1 / 2$ the derivative vanishes almost everywhere.

\section{Quantitative Features of the Solutions}

In order to obtain a more precise picture of how dense shocks are, and in particular to quantitatively verify the conjecture that the inverse Lagrangian function $a(x)$ forms a Devil's staircase, we have performed a much larger simulation with $N=1,600,00$ and with 300 realizations. This permits accurate statistical measurements. In the following, we present the results for both the type $A$ initial data (the first three subsections) and type $B$ initial data (the last subsection).

\subsection{Probability Distribution of Shock Amplitudes}

Let us begin with the distribution of Lagrangian shock intervals $\Delta a$, that is jumps in the inverse Lagrangian function $a(x)$. By (15), this is also the distribution of the 


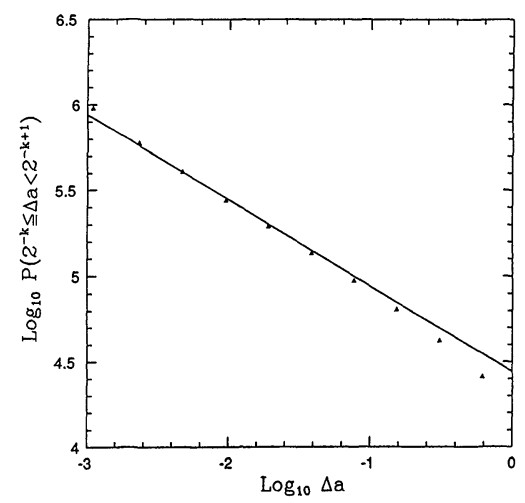

Fig. 5. Cumulative probability of shock amplitudes obtained (from the corresponding Lagrangian shock intervals $\Delta a)$ by octave-binning $\left(2^{-k} \leq \Delta a<2^{-k+1}\right)$. Brownian motion is taken for the initial velocity $(h=1 / 2)$. The straight line is a least square fit which has exponent $-1 / 2$

shock amplitudes. We first consider the case of the Brownian motion as the initial velocity. To facilitate comparisons with analytical results presented in the companion paper, we have calculated the cumulative probability $P(\Delta a)$ to find $\Delta a$ in an octave interval $\left[a_{k}, a_{k+1}\left[\right.\right.$, where $a_{k}=2^{-k}(k=0,1,2, \ldots)$. In Fig. $5, \log P$ is plotted against $\log \Delta a$. We observe that for small Lagrangian shock intervals $(\Delta a \rightarrow 0)$, the probability follows a power law:

$$
P(\Delta a) \propto(\Delta a)^{s} .
$$

The exponent $s$ determined by a least square fit is $s=-0.5 \pm 0.005$ which agrees within one percent with the value obtained in the companion paper. Several other runs have been performed with decreasing mesh-size $\Delta x$, ensuring that the result is representative of the continuous limit $\Delta x \rightarrow 0$.

A similar analysis for the case type $A$ fractional Brownian initial velocity with scaling exponent $h \neq 1 / 2$ shows that cumulative probability of Lagrangian shock intervals also follows the power-law (35). The exponent is now given by $s=-h$ (with high accuracy again). Thus, in all type $A$ cases, the total number of shocks per unit length diverges due to the proliferation of small shocks.

\subsection{Multifractal Analysis for Lagrangian Regular Points}

As shown in the companion paper, the $(\Delta a)^{-1 / 2}$ law for the cumulative probability of Lagrangian shock intervals is connected with another (rigorous) result: the set of Lagrangian regular points has Hausdorff dimension $1 / 2$. Heuristically, this is the dimension one would expect if intervals are removed with a cumulative probability $\propto(\Delta a)^{-1 / 2}$. More detailed information about the set of regular points can be obtained by performing a multifractal analysis of a positive measure concentrated on this set. Such is obviously the case of the measure $\frac{\partial x}{\partial a} d a$ whose density is the derivative (in the sense of distributions) of the Lagrangian function $x(a)$. Indeed, we know that the non-decreasing function $x(a)$ is constant over shock intervals, so that its derivative is non-negative and concentrated on the regular points. 


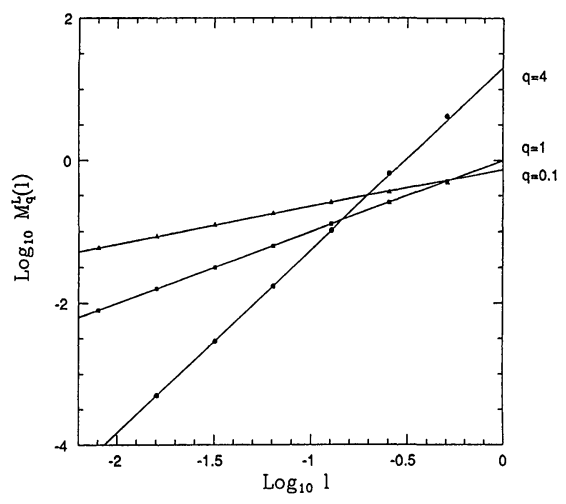

Fig. 6. Moments $M_{q}^{\mathrm{L}}(l)=\left\langle(x(a+l)-x(a))^{q}\right\rangle$ for Brownian initial velocity $(h=1 / 2)$. The straight lines are least square fits over the range of scales where there is power-law behavior: $M_{q}^{\mathrm{L}}(l) \propto l_{q}^{\tau_{q}^{\mathrm{L}}}$

The multifractal analysis involves the following steps ([18, 19]). First, one computes the moments

$$
M_{q}^{\mathrm{L}}(l)=\left\langle\left(\int_{a}^{a+l} \frac{\partial x}{\partial a^{\prime}} d a^{\prime}\right)^{q}\right\rangle=\left\langle(x(a+l)-x(a))^{q}\right\rangle .
$$

The superscript "L" stands for "Lagrangian." In the simulations, the averages are computed by a combination of space and ensemble averaging (over typically 100 realizations of $10^{6}$ space points). In Fig. 6, we show the $l$-dependence of the moments $M_{q}^{\mathrm{L}}(l)$ for several $q$ 's for the case of Brownian initial velocity. It is seen that over two decades in $l$, all moments behave as power laws:

$$
M_{q}^{\mathrm{L}}(l) \propto l^{\tau_{q}^{\mathrm{L}}} .
$$

The exponents $\tau_{q}^{\mathrm{L}}$ plotted in Fig. 7a, can be well fitted empirically by the law $\tau_{q}^{\mathrm{L}} \simeq$ $0.5+0.5 q$. This suggests that there is a single fractal dimension present, namely
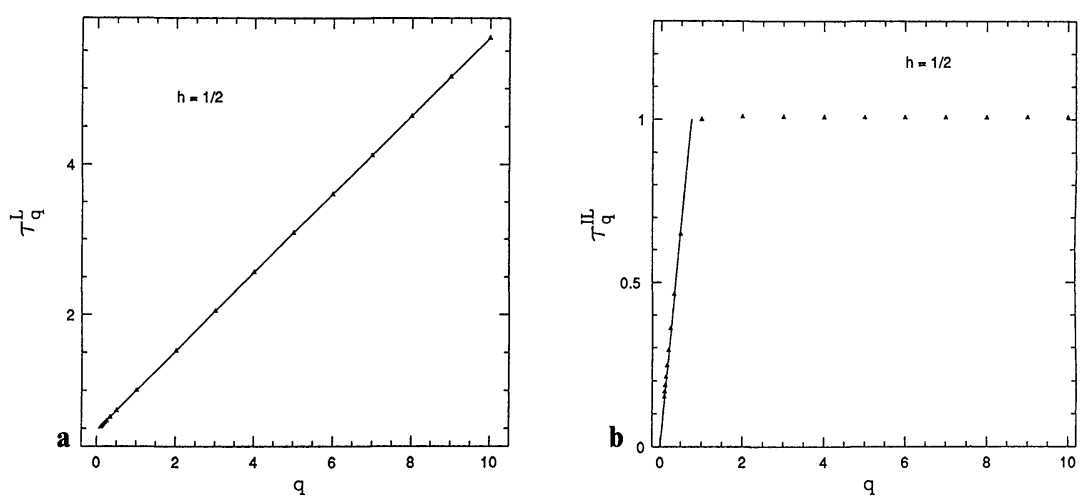

Fig. 7a, b. Dependence of the scaling exponents $\tau_{q}^{\mathrm{L}}(\mathbf{a})$ and $\tau_{q}^{\mathrm{IL}}$ (b) on the order $q$ (see Fig. 6) for Brownian initial velocity $(h=1 / 2)$. Note that in case a the linear dependence on $q$ indicates the existence of a single fractal dimension $(D=h)$. For case $\mathbf{b}$ the presence of a different slope for small and large $q$ 's reflects a bifractality 
$D=1 / 2$. (Recall that the distribution of scaling exponents is the Legendre transform of $\tau_{q}$; cf. [19].) We have thus obtained additional evidence, for the Brownian initial condition, that the set of regular points forms a fractal set of Hausdorff dimension $1 / 2$.

For type $A$ fractional Brownian motion with scaling exponent $h \neq 1 / 2$, analogous simulations (not shown) give the empirical law $\tau_{1}^{\mathrm{L}} \simeq 1-h+h q$. This suggests the following

Conjecture. For initial conditions which are fractional Brownian motion with scaling exponent $0<h<1$, the set of (Lagrangian) regular points has Hausdorff dimension $h$.

\subsection{Multifractal Analysis for (Eulerian) Shock Points}

Eulerian information such as the characterization of the set of shock points [where there is a jump in $a(x)$ ] can be obtained by applying the same multifractal analysis as in the preceding section, but now to the inverse Lagrangian function $a(x)$ rather than to $x(a)$. Indeed, the measure $\frac{\partial a}{\partial x} d x$ is concentrated on shocks. We are thus led to calculate the following set of moments

$$
M_{q}^{\mathrm{IL}}(l)=\left\langle\left(\int_{x}^{x+l} \frac{\partial a}{\partial x^{\prime}} d x^{\prime}\right)^{q}\right\rangle=\left\langle(a(x+l)-a(x))^{q}\right\rangle .
$$

Here, the superscript "IL" stands for "inverse Lagrangian." Again we find that the moments $M_{q}^{\mathrm{IL}}(l)$ scale with $l$ over a suitable range:

$$
M_{q}^{\mathrm{IL}}(l) \propto l^{\tau_{q}^{\mathrm{IL}}} .
$$

However, now the range of scales where the power law behavior is observed is different for $q>1$ and for $q<1$. Specially, for the case of Brownian initial velocity, we obtain the following behavior (see Fig. 7b)

$$
\tau_{q}^{\mathrm{IL}}= \begin{cases}\alpha q & 0<q<q^{*}<1 \\ 1 & q^{*}<q\end{cases}
$$

with $\alpha \approx 1.33$. A similar numerical experiment, performed with fractional Brownian initial velocity $(h=0.7)$, gives $\alpha \approx 1.30$. In both cases, $q^{*} \approx 0.5$. We do not attach too much significance to this value, because the crossover from one to the other scaling régime was not studied in a great detail.

The result above suggests that we have a bifractal structure as discussed in [20]: singularities of exponent zero on a set of dimension zero and singularities of exponent $\alpha$ [see (40)] on a set of dimension one. The former could be the contributions of strong isolated shocks. The latter can be obtained by a heuristic argument based on (31). If we assume that $a$ and $a^{\prime}$ are close, we have

$$
u_{0}(a)-u_{0}\left(a^{\prime}\right)=O\left(\left|a-a^{\prime}\right|^{h}\right) .
$$

Neglecting the first term compared to the second in the r.h.s. of (31), and inverting (if legitimate), we obtain

$$
a-a^{\prime}=O\left(\left|x-x^{\prime}\right|^{1 / h}\right) .
$$


The substantial discrepancy between $1 / h$ and the measured value $\alpha$ may be due to a slow convergence of small- $q$ moments towards their true asymptotic value.

A bifractality similar to the one observed here exists in the standard Devils staircaise for the Cantor set. If one takes away a fraction $\sigma$ at every step ( $\sigma$ being $\frac{1}{3}$ in the middle third Cantor set) it is easy to see by explicit computation that

$$
\begin{aligned}
\tau_{q}^{\mathrm{L}} & =1+(q-1) D_{0} \\
\tau_{q}^{\mathrm{IL}}=\left\{\begin{array}{cc}
\frac{q}{D_{0}} & q<D_{0} \\
1 & q>D_{0}
\end{array}\right\} & \text { (Cantor staircase) }
\end{aligned}
$$

where $D_{0}$ is the Hausdorff dimension (here $-\log 2 / \log \sigma$ ) of the set. For simple generalizations, such as asymmetric bisection ratios, the function $\tau_{q}^{\mathrm{L}}$ depends on the details of the construction procedure [19]. The analog of the measure $\partial x / \partial a$, concentrated on Lagrangian regular points, is then multifractal. It can be shown that the low- $q$ branch $\left(q<D_{0}\right)$ of $\tau_{q}^{\mathrm{IL}}$ is related to the high- $q$ branch $(q>0)$ of $\tau_{q}^{\mathrm{L}}$ through

$$
\tau_{q}^{\mathrm{IL}}=1-q^{\prime}, \quad q=1-\tau_{q^{\prime}}^{\mathrm{L}} .
$$

Finally, we mention that we have also measured standard Eulerian statistical properties of the velocity field, such as the structure functions

$$
\left\langle(u(x+l)-u(x))^{p}\right\rangle
$$

for $p \geq 1$. These calculations were done only for the case of Brownian initial velocity ( $h=1 / 2$ ). We found that, for large l's the structure functions scale just as the initial conditions, namely $\propto l^{p / 2}$, whereas, for small l's they scale just as the structure functions of Burgers' equation with smooth initial conditions [20], namely $\propto l^{1}$ for all $p \geq 1$. The former is probably due to the fact that the large-scale properties of the solution for $t=1$ are deducible [by (21)] from the properties for short times of the solution for scales order one. The latter is explained by observing that there is a dominant contribution to structure functions of order $p \geq 1$ coming from strong shocks, which are well separated. Further studies of structure functions for $h \neq 1 / 2$ will be reported elsewhere.

\subsection{Initial Conditions of Type B}

We have repeated all of the above described analysis for initial conditions of type $B$ for which $-1<h<0$. It is now the initial potential function, rather than the velocity, which is a fractional Brownian motion. For the case of the white noise $(h=-1 / 2)$, Fig. 8 indicates that the cumulative probability of Lagrangian shock intervals is still approximately given, as for type $A$, by a power law:

$$
P(\Delta a) \propto(\Delta a)^{s} .
$$

A least square fit indicates $s \simeq 0.5$. A comparison between Fig. 8 and Fig. 5 (type $A ; h=1 / 2$ ) shows that the scaling is not as clean as before. Still, the fact that the exponent $s$ is now clearly positive shows that there is only a finite number (per unit 


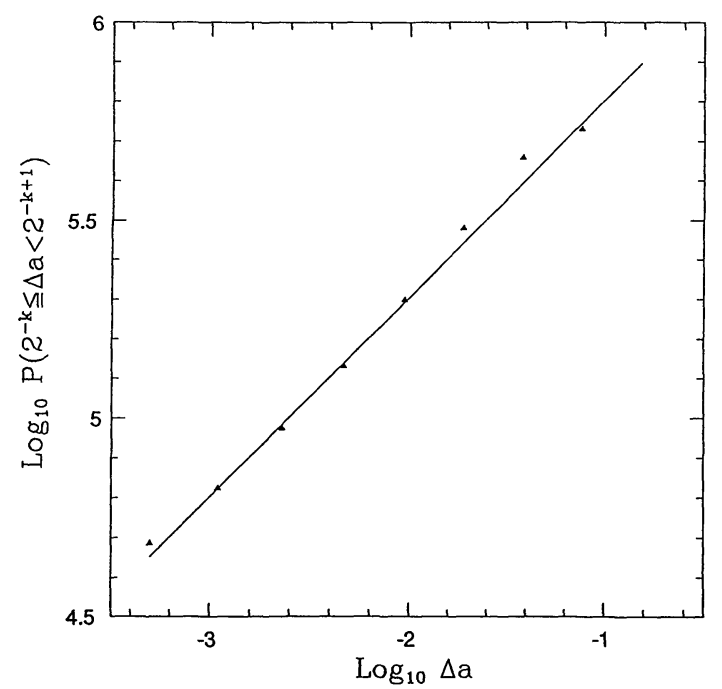

Fig. 8. Cumulative probability of shock amplitudes obtained (from corresponding Lagrangian shock intervals $\Delta a)$ by octave-binning $\left(2^{-k} \leq \Delta a<2^{-k+1}\right)$. White noise is taken for the initial velocity $(h=-1 / 2)$. The straight line is a least square fit which has exponent $1 / 2$
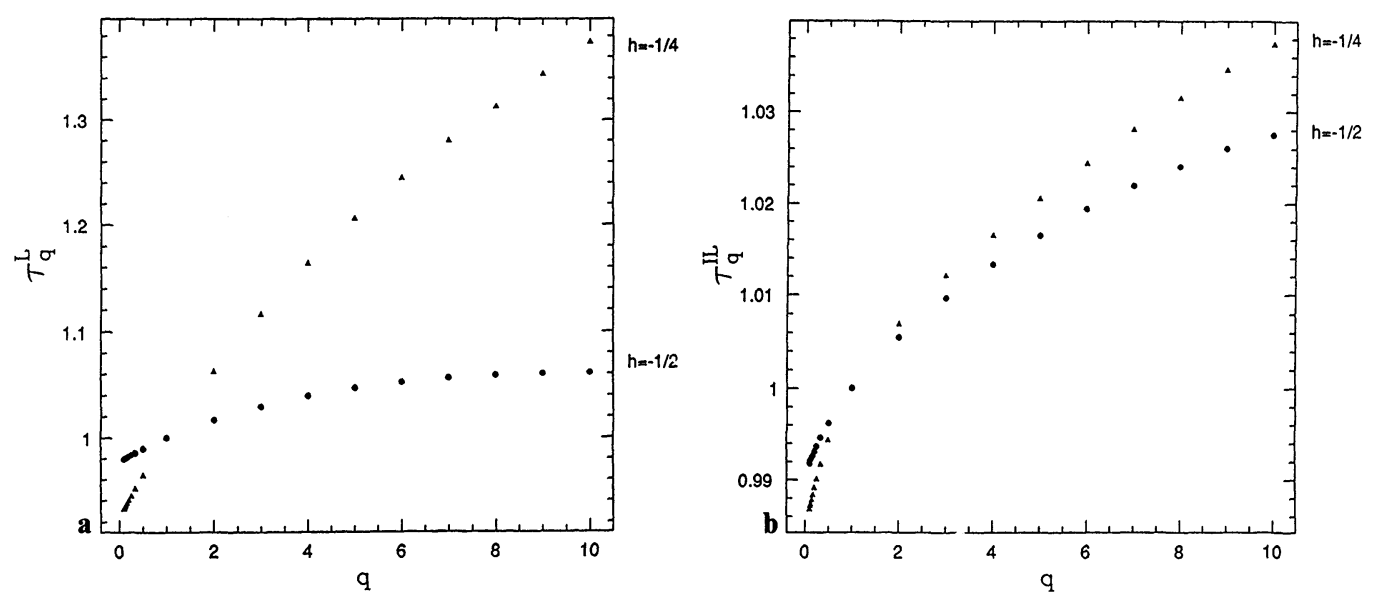

Fig. 9a, b. Scaling exponents $\tau_{q}^{\mathrm{L}}$ (a) and $\tau_{q}^{\mathrm{IL}}$ (b) obtained as in Fig. 7 but with initial velocity of type $B . h=-1 / 2$ : circles; $h=-1 / 4$ : triangles

length) of small Lagrangian shock intervals. Thus, no Cantor-like set can be obtained by removing such intervals, i.e. for the Lagrangian regular points.

We now turn to the multifractal analysis for the solution of type $B$ initial conditions. We again find that there is scaling for both the moments $M_{q}^{\mathrm{L}}(l)$ and $M_{q}^{\mathrm{IL}}(l)$. The scaling exponents $\tau_{q}^{\mathrm{L}}$ and $\tau_{q}^{\mathrm{IL}}$, shown in Fig. $9 \mathrm{a}$ and $9 \mathrm{~b}$ (for $h=-1 / 2$ and $h=-1 / 4$ ), are not straight lines: they both display curvature. This could be taken to suggest that the measure $\frac{\partial x}{\partial a} d a$ and the measure $\frac{\partial a}{\partial x} d x$ have both non-trivial multifractal behavior for type $B$ initial conditions. It is likely, however, that such weak multifractality is 
a numerical artefact. Actually, for the white noise initial condition $(h=-1 / 2)$, the large- $q$ behavior of both $\tau_{q}^{\mathrm{L}}$ and $\tau_{q}^{\mathrm{IL}}$ suggests a straight line with slope zero. (The vertical scales are very much stretched, especially for Fig. 9b.) This is consistent with our previous conjecture that both Eulerian shock points and Lagrangian regular points are isolated.

\section{Conclusion}

We begin by summarizing our main quantitative results. They concern (i) the cumulative probability $P(\Delta a)$ of Lagrangian shock intervals $\Delta a$, (ii) the moments $M_{q}^{\mathrm{L}}(l)$ of increments of the Lagrangian function $x(a)$ over a distance $l$, (iii) the moments $M_{q}^{\mathrm{IL}}(l)$ of increments of the inverse Lagrangian function $a(x)$. (These increments are equal to the integrals of the mass density when the latter is initially assumed uniform.) We have found that $P(\Delta a)$ behaves for small $\Delta a$ as $(\Delta a)^{-h}$. This result holds for type $A(0<h<1)$ with very good accuracy, and also for type $B(-1<h<0)$, but not so accurately. For type $A$, the multifractal analysis indicates the following: the measure $\partial x / \partial a$, concentrated on Lagrangian regular points, is monofractal with a dimension equal to the scaling exponent $h$; the measure $\partial a / \partial x$ (the mass density) is consistent with a bifractal description involving dimensions 0 and 1 with respective scaling exponents 0 and $1 / \mathrm{h}$. (Precisely the same result holds for Cantor-like constructions, as explained in Sect.6.3.) For type $B$, our multifractal numerical results may not be fully reliable. The indications are that both the Eulerian shock points and the Lagrangian regular points are isolated.

We now turn to a different matter. As we shall briefly show, the results established in this paper, for rather special non-smooth initial conditions of Brownian type, have actually implications for a large class of smooth random initial conditions, provided very large times and very large scales are considered. Let us explain this for the white noise (a particular type $B$ ) initial condition. Consider the universality class of initial conditions having all of the following properties, which are expressed in terms of the initial velocity: $u_{0}(x)$ is random homogeneous and smooth (infinitely many derivatives), has zero mean value and finite variance, is mixing (correlations decrease with separation) and has a correlation function $\Gamma\left(x-x^{\prime}\right)=\left\langle u_{0}(x) u_{0}\left(x^{\prime}\right)\right\rangle$ such that $+\infty$

$\int_{-\infty}^{+\infty} \Gamma(x) d x=D>0 .{ }^{1}$ These assumptions imply by a central limit argument that

$$
\frac{1}{\varepsilon} \psi_{0}\left(\frac{y}{\varepsilon^{2}}\right)=-\frac{1}{\varepsilon} \int_{0}^{\frac{y}{\varepsilon^{2}}} u_{0}\left(y^{\prime}\right) d y^{\prime}
$$

tends for $\varepsilon \rightarrow 0$ to the Brownian motion function (scaled by a factor $D^{1 / 2}$ ). Consider now the solution to Burgers equations given by (6). Simple rescaling gives the following relation:

$$
\frac{1}{\varepsilon} \psi\left(\frac{x}{\varepsilon^{2}}, \frac{t}{\varepsilon^{5}}\right)=\max _{y}\left[\frac{1}{\varepsilon} \psi_{0}\left(\frac{y}{\varepsilon^{2}}\right)-\frac{(x-y)^{2}}{2 t}\right] .
$$

It thus follows that, for $\varepsilon \rightarrow 0$, the rescaled solution tends to the solution corresponding to an initial velocity which is the (negative) derivative of the Brownian

\footnotetext{
1 Other more technical assumptions may be needed for a rigorous proof
} 
motion function, namely white noise. Thus, the long-time large-scale behavior can be obtained from the solution of Burgers' equation with non-smooth initial velocity of type $B$ (with $h=-1 / 2$ ). Burgers himself was probably aware of this, since in his book he uses diffusion techniques to study the long-time behavior of the solution to his equation for random initial conditions having $D \neq 0$ (see Sect. 9 of [2]). We observe that similar universality classes are associated to any type $A$ or type $B$ solutions. It is easily seen that the universality class to which a given smooth initial random velocity field belongs depends only on the behavior of the energy spectrum $E_{0}(k)$ of the initial velocity at small wavenumbers. If $E_{0}(k) \propto|k|^{-1-2 h}$ as $k \rightarrow 0$, the long-time large-scale behavior will be as for type $A$ or type $B$ solutions having scaling exponent $h$.

Let us finally mention that the present study can be extended to the multidimensional Burgers equation, which reads, in terms of the potential function $\psi(\mathbf{r}, t)$ :

$$
\partial_{t} \psi=\frac{1}{2}|\nabla \psi|^{2}+\nu \nabla^{2} \psi
$$

The Hopf-Cole transformation and the Legendre transformation method (for $\nu \rightarrow 0$ ) are readily extended, but the theoretical analysis and the numerical experiments become considerably more involved. Early results from high resolution two-dimensional simulations indicate that scaling properties similar to those of the one-dimensional case are found [21].

Acknowledgements. We are grateful to Y. Sinai for many discussions. We have also benefited from discussions with M. Hénon. This work was supported by AFOSR (90-0124) and DARPA (N0001486-K-0759) (Z.S.S.), by the EEC (SC1-0212-C), DRET (90/1444) and by the Göran Gustavsson Foundation (E.A.).

\section{References}

1. Burgers, J.M.: On the application of statistical mechanics to the theory of turbulent fluid motion. Proc. Roy. Neth. Acad. Soc. 32, 414, 643, 818 (1929)

2. Burgers, J.M.: The nonlinear diffusion equation. Dordrecht: D. Reidel 1974

3. Hopf, E.: The partial differential equation $u_{t}+u u_{x}=u_{x x}$. Commun. Pure Appl. Math. 3, 201 (1950)

4. Cole, J.D.: On a quasi-linear parabolic equation occurring in aerodynamics. Quart. Appl. Math. 9, 225 (1951)

5. Zel'dovich, Ya.B.: Gravitational instability: An approximate theory for large density perturbations. Astro. Astrophys. 5, 84 (1970)

6. Kardar, M., Parisi, G., Zhang, Y.C.: Dynamical scaling of growing interfaces. Phys. Rev. Lett. 56, 889 (1986)

7. Kuramoto, Y.: Chemical oscillations, waves and turbulence. Berlin, Heidelberg, New York: Springer 1985

8. Musha, T., Kosugi, Y., Matsumoto, G., Suzuki, M.: Modulation of the time relation of action potential impulses propagating along an axon. IEEE Trans. Biomedical Eng. BME-28, 616-623 (1981)

9. Fournier, J.D., Frisch, U.: L'équation de Burgers déterministe et statistique. J. Méc. Théor. Appl. 2, 699 (1983)

10. Kida, S.: Asymptotic properties of Burgers turbulence. J. Fluid Mech. 93, 337 (1979)

11. Gurbatov, S.N., Saichev, A.I.: Degeneracy of one-dimensional acoustic turbulence at large Reynolds numbers. Sov. Phys. JETP. 53, 347 (1981)

12. Gurbatov, S.N., Saichev, A.I., Shandarin, S.F.: The large-scale structure of the Universe in the frame of the model equation of non-linear diffusion. Mon. Not. R. Astro. Soc. 236, 385-402 (1989)

13. Shandarin, S.F., Zel'dovich, Ya.B.: The large-scale structure of the Universe: Turbulence, intermittency, structures in a self-gravitating medium. Rev. Mod. Phys. 61, 185 (1989) 
14. Weinberg, D., Gunn, J.: Large-scale structure and the adhesion approximation. Mon. Not. R. Astro. Soc. 247, 260-286 (1990)

15. Williams, B.G., Heavens, A.F., Peacock, J.A., Shandarin, S.F.: Exact hierarchical clustering in one dimension. Monthly Notices R. Astron. Soc. 250, 458-476 (1991)

16. Gurbatov, S.N., Malakhov, A.N., Saichev, A.I. Nonlinear random waves and turbulence in nondispersive media: waves, rays, particles. Manchester University Press 1991

17. Sinai, Ya.G.: Statistics of shocks in solutions of the inviscid Burgers equation. Commun. Math. Phys. 148, 601 (1992)

18. Parisi, G., Frisch, U.: On the singularity structure of fully developed turbulence. In: Turbulence and predictability in geophysical fluid dynamics. Proc. Intern. School of Physics "E. Fermi", 1983, Varenna, Italy, Ghil, M., Benzi, R., Parisi, G. (eds.), p. 84, North-Holland 1985

19. Halsey, T.C., Jensen, M.H., Kadanoff, L.P., Procaccia, I., Shraiman, B.I.: Fractal measures and their singularities: the characterization of strange sets. Phys. Rev. A33, 1141 (1986)

20. Aurell, E., Frisch, U., Lutsko, J., Vergassola, M.: On the multifractal properties of the energy dissipation derived from turbulence data. J. Fluid Mech. 238, 467 (1992)

21. Vergassola, M., 1991: Private communication

Communicated by Ya. G. Sinai 
